\title{
CanPrevent: a telephone-delivered intervention to reduce multiple behavioural risk factors for colorectal cancer
}

\author{
Anna L Hawkes ${ }^{1,2 *}$, Tania A Patrao ${ }^{2}$, Anita Green ${ }^{3}$ and Joanne F Aitken ${ }^{2}$
}

\begin{abstract}
Background: This pilot study aimed to test the acceptability and short-term effectiveness of a telephone-delivered multiple health behaviour change intervention for relatives of colorectal cancer survivors.

Methods: A community-based sample of 22 first-degree relatives of colorectal cancer survivors were recruited via a media release. Data were collected at baseline and at six weeks (post-intervention). Outcome measures included health behaviours (physical activity, television viewing, diet, alcohol, body mass index, waist circumference and smoking), health-related quality of life (Short Form-36) and perceived colorectal cancer risk. Intervention satisfaction levels were also measured. The intervention included six telephone health coaching sessions, a participant handbook and a pedometer. It focused on behavioural risk factors for colorectal cancer [physical activity, diet (red and processed meat consumption, fruit and vegetable intake), alcohol, weight management and smoking], and colorectal cancer risk.
\end{abstract}

Results: From baseline to six weeks, improvements were observed for minutes moderate-vigorous physical activity (150.7 minutes), processed meat intake $(-1.2$ serves/week), vegetable intake (1 serve/day), alcohol intake ( -0.4 standard drinks/day), body mass index $(-1.4 \mathrm{~kg} / \mathrm{m} 2)$, and waist circumference $(-5.1 \mathrm{~cm})$. Improvements were also observed for physical (3.3) and mental (4.4) health-related quality of life. Further, compared with baseline, participants were more likely to meet Australian recommendations post-intervention for: moderate-vigorous physical activity ( 27.3 vs $59.1 \%)$; fruit intake (68.2 vs $81.8 \%)$; vegetable intake (4.6 vs 18.2\%); alcohol consumption (59.1 vs 72.7\%); body mass index (31.8 vs $45.5 \%$ ) and waist circumference (18.2 vs $27.3 \%$ ). At six weeks participants were more likely to believe a diagnosis of CRC was related to family history, and there was a decrease in their perceived risk of developing CRC in their lifetime following participation in CanPrevent. The intervention retention rate was $100 \%$, participants reported that it was highly acceptable and they would recommend it to others at risk of colorectal cancer.

Conclusions: Positive behaviour change achieved through this intervention approach has the potential to impact on the progression of CRC and other cancers or chronic diseases. A large scale randomised controlled trial is required to confirm the positive results of this acceptability and short-term effectiveness study.

Trial registration: ACTRN12612000516886

Keywords: Colorectal cancer, Multiple health behaviour change intervention, Lifestyle, Physical activity, Telephone, Prevention, Family history

\footnotetext{
* Correspondence: Anna.Hawkes@gmail.com

${ }^{1}$ School of Public Health and Social Work, Queensland University of

Technology, Victoria Park Road, Kelvin Grove, Brisbane, Queensland 4059,

Australia

${ }^{2}$ Viertel Centre for Research in Cancer Control, Cancer Council Queensland,

PO Box 201, Spring Hill, Brisbane, Queensland 4004, Australia

Full list of author information is available at the end of the article
}

\section{Biomed Central}

(c) 2012 Hawkes et al.; licensee BioMed Central Ltd. This is an Open Access article distributed under the terms of the Creative Commons Attribution License (http://creativecommons.org/licenses/by/2.0), which permits unrestricted use, distribution, and reproduction in any medium, provided the original work is properly cited. 


\section{Background}

Colorectal cancer (CRC) is one of the leading causes of cancer morbidity and mortality in the industrialized world [1]. Most cases (93\%) occur in persons aged 50 years or more [2] and it often co-exists with other chronic diseases related to health behaviours including obesity, type 2 diabetes mellitus and cardiovascular disease $[3,4]$. Behavioural risk factors including physical inactivity [5], diet [6-8] and obesity [9-11] play a pivotal role in the aetiology of CRC, and it has been estimated that at least $70 \%$ of CRC may be prevented with moderate behavioural changes [12]. In particular, reductions in the consumption of alcohol and red and processed meat, weight loss and increased levels of physical activity may translate into significant reductions in the incidence of CRC [12]. Importantly, these lifestyle changes also decrease risk of other cancers as well as type 2 diabetes mellitus and cardiovascular disease $[13,14]$, therefore behavioural improvements result in overall health benefits.

Individuals with a family history of CRC have a significantly elevated risk of developing CRC [15]. Epidemiological studies indicate that first degree relatives of CRC survivors (parents, siblings, or offspring) have a 1.6 to 8times higher life time risk of CRC than those without a family history, the strength of the relationship varying according to age at diagnosis in the index case, type of relative, and the number of relatives affected [15]. Furthermore, a combination of familial predisposition and unhealthy behaviours increases risk of CRC considerably [16].

Despite the evidence, research has shown that most people are unaware of the association between behavioural risk factors and CRC risk [17], and individuals identified at high risk of CRC do not generally make voluntary behavioural changes [18]. One study found that first degree relatives of CRC survivors attributed their risk of CRC to physiology (27\%) or family history (25\%), whilst only $16 \%$ believed behavioural risk factors were of importance [19]. As such, it is important to educate people about the importance of their health behaviours and to support those at high risk of CRC to make improvements to reduce their risk of the disease. To our knowledge, there are no programs routinely available to support individuals considered at increased risk of CRC. The absence of specific programs for this population group remains a missed opportunity as national policy supports cancer reduction and the evidence suggests that behaviour change programs targeting high risk groups may be more effective than those targeting the population at large $[20,21]$.

There is also a paucity of research investigating educational or supportive interventions specifically for those at high risk of CRC with, to our knowledge, just two published studies in the field specifically targeting those with colorectal adenomas [20,21]. Bowel health to better health was a trial of a three month minimal contact intervention (one face to face session followed by three personalised mailings; $n=74$ ). The intervention included lifestyle advice, goal setting and social support to promote increases in physical activity, fibre, fruit and vegetable intake. However, the study was limited by a low response rate $(51 \%)$, and intervention effects were observed for fibre intake alone [20]. Project PREVENT was a trial of a tele-based counselling intervention based on Social Cognitive Theory [22] to improve multiple risk factors (red meat, fruit, vegetable, multivitamin and alcohol intake, smoking, and physical activity; $n=1247$ ). Intervention effects were observed for multiple risk factors (including multivitamin and red meat intake), and intervention participants tended to have a lower rate of regression in their levels of physical activity than usual care participants. However, there were no direct intervention effects on smoking, alcohol, fruit or vegetable intake, and the study was limited by the inclusion of participants who were highly educated [21].

Behavioural risk factors for CRC are interrelated in terms of the psychological, social and environmental factors that reinforce them [23] (for example, those who eat high-fat diets are more likely to be sedentary and to be cigarette smokers) $[22,24,25]$. Also, previous investigations $[24,26]$ have shown that change in one behavioural risk factor may serve as a stimulus or gateway for change in other health behaviours. However, few CRC studies have intervened on multiple behaviours simultaneously. It represents a challenge from an intervention perspective, but provides an important opportunity to maximise the potency of cancer prevention interventions as the complex and multifactorial process of carcinogenesis suggests that several behavioural changes may be needed to significantly reduce risk. Thus multiple risk factor interventions warrant further study [21].

Theory-based behavioural interventions have been shown to be most effective and Social Cognitive Theory is widely used [22,27]. In contrast, the CanPrevent intervention used specific strategies from Acceptance Commitment Therapy (ACT), which is an empirically based third generation cognitive behavioural approach that uses acceptance and mindfulness strategies, and commitment and behaviour change strategies to produce psychological flexibility: the ability to defuse from difficult thoughts and accept difficult feelings while persisting in values-based action [28-31]. This provided an alternative to existing intervention approaches by overcoming internal barriers to making lifestyle improvements by emphasizing the role of emotions and thoughts in the maintenance of good self-management of lifestyle factors [32]. To date, ACT interventions have been successfully used to enhance quality of life and promote positive 
lifestyle behaviours for a range of health conditions (chronic pain [33], diabetes [34], epilepsy [35], smoking [36], and obesity or weight management [37-39]) but this approach has not previously been used for those at high risk of CRC.

Previous researchers have investigated a range of delivery modes for behavioural interventions (face to face, telephone, internet and paper-based delivery) and telephone-delivered interventions have been shown to be highly acceptable [40], improve behavioural outcomes in the short term $[41,42]$ for cancer survivors, and there is a solid evidence base supporting the efficacy of telephone based interventions for physical activity and dietary behaviour change [43,44]. Importantly, in Australia, approximately $96 \%$ of the population live in a household with at least one telephone connection, hence this approach appeared viable for the current study [45].

This is the first pilot study of the acceptability and short-term effectiveness of a novel theory-based telephone-delivered multiple risk factor intervention to improve behavioural risk factors, health-related quality of life (HRQoL) and perceived risk of CRC for first degree relatives of CRC survivors.

\section{Methods}

\section{Participants}

From February to March 2011 (eight weeks) adults resident in Queensland, Australia who were a first degree relative of someone with a confirmed diagnosis of primary CRC (C18-C20, C218) were sourced through Queensland based media advertisements (print, radio, internet). Eligibility criteria included: (i) ability to understand and provide written informed consent in English; (ii) no current or previous diagnosis of CRC; (iii) no medical conditions that would limit adherence to an unsupervised lifestyle program; (iv) a telephone; and (v) those who had at least one poor health behaviour consistent with Australian recommendations [46-49] [do not achieve $\geq 150$ minutes moderate-vigorous physical activity per week; or do not adhere to a healthy diet (indicated by $>4$ serves red meat/week, or $<2$ serves fruit per day, or $<5$ serves vegetables per day); or consume $>2$ standard drinks per day; or are overweight (body mass index or BMI $\geq 25$ ). Participants were screened for eligibility prior to recruitment.

\section{Data collection}

Data were collected at baseline (pre-intervention) and at six weeks (post-intervention) by dedicated computer assisted telephone interviewers using measures that have previously been used in longitudinal studies over the phone [43,50-52]. Information was collected on outcomes targeted by the intervention including: behavioural risk factors [physical activity, television (TV) viewing, diet, alcohol, BMI, waist circumference and smoking], generic HRQoL and perceived CRC risk. At the completion of the intervention, participants were mailed a self-reported survey to assess satisfaction with the intervention.

\section{Physical activity}

We used a modified version of the leisure score index of the Godin Leisure-Time Exercise Questionnaire that has been shown to be a reliable and valid self-report measure of physical activity. The leisure score index contains three questions that assess the average frequency of mild, moderate, and strenuous exercise during free time in a typical week. The modified version of the leisure score index also provides average duration of physical activity [53]. Participants were categorised in to $\geq 150$ minutes/week moderate to vigorous physical activity or $<150$ minutes/week moderate-vigorous physical activity consistent with Australian recommendations [46].

\section{TV viewing}

Within epidemiological and health behaviour research, measurement of adults' sedentary behaviour has often focused on TV viewing, one of the most frequently reported leisure-time activities [54]. Participants provided an estimate of the total time spent watching TV, on an average day, over the past month. Self-reported $\mathrm{TV}$ viewing has been shown to be a reasonably reliable and valid measure for adults [55].

\section{Diet and alcohol}

We used brief questions about diet and alcohol behaviour (red meat, processed meat, vegetable, fruit, alcohol intake) based on the Cancer Council New South Wales validated and commonly used items for assessing diet and alcohol in cancer patients [56]. Consistent with national recommendations [57] participants were categorised in to: (i) red meat intake $\leq 4$ serves/week or $>4$ serves/week; (ii) vegetable intake $\geq 5$ serves/week or $<5$ serves/week; (iii) fruit intake $\geq 2$ serves/week or $<2$ serves/week; and (iv) alcohol consumption $\leq 2$ standard drinks/week or $>2$ standard drinks/week. There are no specific national recommendations for processed meat intake ('avoid processed meats') [57].

\section{Smoking}

We used brief validated questions about smoking behaviour developed by the Cancer Council New South Wales for assessing smoking behaviour in cancer patients [56].

\section{BMI}

Self-reported height and weight were recorded and they were categorised as healthy weight (BMI $18.5-24.9 \mathrm{~kg} /$ $\mathrm{m} 2$ ), overweight (BMI $25.0-29.9 \mathrm{~kg} / \mathrm{m} 2$ ), or obese (BMI $\geq 30.0 \mathrm{~kg} / \mathrm{m} 2$ ) consistent with national recommendations 
[58]. Participants were given an instruction sheet on how to accurately take measurements and they provided selfreported height and weight which has been shown to provide accurate results in previous telephone-delivered intervention trials $[43,44,50]$.

\section{Waist circumference}

Participants were provided with tape measures and an instruction sheet on self measurement of waist circumference $(\mathrm{cm})$ and prior to the telephone interview, as previous investigators have demonstrated a high correlation between self-reported and technician-recorded waist circumference in males and females [59].

Participants were classified as low risk $(\leq 94 \mathrm{~cm}$ men, $\leq 80 \mathrm{~cm}$ women), increased risk $(>94 \mathrm{~cm}$ men, $>80 \mathrm{~cm}$ women) or greatly increased risk $(>102 \mathrm{~cm}$ men, $>88 \mathrm{~cm}$ women) consistent with national recommendations [60].

\section{HRQoL}

We used the Short Form-36 (SF-36), a widely used measure that has published norms for the Australian general population [61,62]. The SF-36 provides a physical- and mental- HRQoL summary measure suitable to measure the impact of the intervention on patients' wellbeing. It also provides eight sub-scales including: physical functioning, role-physical, bodily pain, general health, vitality, social functioning, role-emotional and mental health $[61,62]$.

\section{CRC screening and perceived risk of CRC}

Participants were asked whether they had ever been screened for CRC and to provide the approximate date and screening test used, they were also asked whether they intended to be screened in the future. In addition, participants were asked about their perceived risk of CRC using a modified version of brief validated questions [19] ['What proportion of people in the general population do you believe are diagnosed with CRC due to family history?', 'What do you think your chances are of ever developing CRC in your lifetime?' (scored 0-100\%)].

\section{Intervention satisfaction}

Participants were asked about their satisfaction (scored on a four point likert scale from 'highly unsatisfied, 'unsatisfied', 'satisfied' to 'highly satisfied') with the CanPrevent program overall, the health coaches and the CanPrevent Handbook. Participants were also asked to indicate (yes, no) whether they would recommend the intervention to others at risk of CRC.

\section{Intervention}

The intervention included six evidence-based telephone health coaching sessions delivered by study-trained health professionals ('health coaches'), and a participant handbook, worksheets and a Yamax SW700 Multifunction DigiWalker pedometer. Health coaches had tertiary qualifications in nursing, psychology or health promotion. They received six weeks of study-specific training in ACT, behavioural models of health and illness and behaviour change, and Australian recommendations for health behaviours. The program focused on supporting participants to make positive lifestyle changes (physical activity, diet, weight management, alcohol and smoking) and to uptake CRC screening consistent with national guidelines [4649,57,63,64]. The intervention included an evidence-based approach with strategies drawn from the core components of ACT [28,31]. ACT is an empirically based third generation cognitive behavioural approach that uses acceptance and mindfulness strategies, and commitment and behaviour change strategies to produce psychological flexibility: the ability to defuse from difficult thoughts and accept difficult feelings while persisting in values-based action. Psychological flexibility was established through six core ACT processes: acceptance, cognitive defusion (changing our relationship with thoughts), being present, self-as-context, values and committed action [31]. The approach explicitly taught strategies designed to increase tolerance in the service of goal-directed behaviour, such as healthy eating and physical activity. Importantly, we were not trialling a psychotherapeutic intervention; rather ACT strategies were used to enhance positive lifestyle behaviours.

Intervention strategies included motivational interviewing, problem solving, action planning and goal setting, as well as reviewing and ongoing monitoring to enhance lifestyle change. The health coaching sessions ran over six weeks for one hour each (an introductory session followed by four weekly and one fortnightly session). Intervention sessions included: (i) An introduction session covering: motivation, expectations, and understanding of CanPrevent; family history and personal circumstances; the role of the health coach; using the CanPrevent Handbook; using the pedometer; and participants were asked to complete a worksheet called "My Healthiest Life Wish List" detailing their health-related values, and to commence tracking their lifestyle factors (physical activity, diet, alcohol, BMI, smoking); (ii) Sessions one to four covered: values and mindfulness, and action planning and goal setting to improve lifestyle factors; (iii) Session five covered: reviewing the previous sessions and action planning and goal setting beyond CanPrevent. Telephone sessions were at no cost to the study participant.

The participant handbook included educational information on health behaviours and the core components of ACT, as well as tracking and monitoring tables for health behaviour change. Using the pedometer, participants were encouraged to achieve 10,000 steps/day as the recommended goal $[46,65]$, and to track and monitor their steps throughout the intervention. 
The study protocol was approved by the University of Queensland Behavioural Social Sciences Ethics Review Committee. To ensure fidelity of intervention delivery, the intervention protocol was detailed in a manual, all sessions were scripted and all intervention calls were audio-taped and reviewed against a session checklist based on the objectives for each session. The health coaches also met with the lead investigator with expertise in behaviour change for bi-weekly supervision sessions.

\section{Statistical analyses}

Data from the telephone interviews were checked for out-of-range or inconsistent data. Descriptive statistics [n (\%) and mean (standard deviation or SD)] were used to describe variables. T-tests were used to measure the change from baseline to six weeks in behavioural variables and HRQoL. Mean differences (95\% confidence interval or $\mathrm{CI}$ ) with corresponding $\mathrm{p}$-values have been presented. From baseline to six weeks, $\mathrm{Chi}^{2}$ tests were used to compare the proportion of participants meeting the national recommendations for behavioural variables and to compare perceived risk of CRC, corresponding $\mathrm{p}$ values are presented. Statistical significance was set at $\mathrm{p} \leq 0.05$. All analyses were conducted using Stata statistical software (Statacorp, College Station, TX, USA).

\section{Results}

The first 28 potential participants were screened for eligibility and 22 eligible participants (79\%) were recruited to the study. We continued to receive expressions of interest from potential participants (total $n=61$ ) over the study recruitment period, however sample size was not increased as we had reached our required sample size for a small pilot study to provide useful information about the acceptability and short-term effectiveness of the intervention. Non-participants were sent a covering letter and standard Cancer Council Queensland resources on reducing risk of cancer. Reasons for ineligibility included: previous diagnosis of $\mathrm{CRC}(\mathrm{n}=2)$, not a first degree relative of a CRC survivor $(n=2)$, or meeting the Australian recommendations for health behaviours $(n=2)$. All 22 participants received 6 health coaching sessions over the 6 week intervention period. Baseline demographic variables are presented in Table 1 . In brief, participants were middle aged (mean age $=47.3 \mathrm{yrs}$ ), and the majority $(82 \%)$ were female, born in Australia (91\%), married or in a de-facto relationship (73\%) and had completed high school (96\%). All participants had at least 1 first degree relative diagnosed with CRC with 4 participants (18\%) having more than 1 first degree relative with CRC.

\section{Health behaviours}

Mean change in health behaviours from baseline to 6 weeks for minutes moderate-vigorous physical activity,
Table 1 Demographic variables

\begin{tabular}{ll}
\hline Variable & $\mathbf{n = 2 2}$ \\
\hline Age, mean (SD) & $47.3(13.4)$ \\
Gender, n (\%) female & $18(81.8)$ \\
Born in Australia, n (\%) & $20(90.9)$ \\
Aboriginal/Torres Strait Islander, n (\%) & 0 \\
English speaking, n (\%) & $22(100)$ \\
Married/De-facto, n (\%) & $16(72.7)$ \\
Completed at least high school, $\mathrm{n}(\%)$ & $21(95.5)$ \\
Employed, $\mathrm{n}(\%)$ & $13(59.1)$ \\
Private Health Insurance, $\mathrm{n}(\%)$ & $19(86.4)$ \\
Household income, $\mathrm{n}(\%)$ & \\
- < $\$ 25,000$ & $4(18.2)$ \\
- \$25,000-\$40,000 & $1(4.6)$ \\
- \$40,000-\$65,000 & $6(27.3)$ \\
- \$65,000-\$100,000 & $5(22.7)$ \\
- > \$100,000 & $1(4.6)$ \\
Number of First Degree Relatives >1, n (\%) & $4(21.1)$ \\
\hline
\end{tabular}

TV viewing, diet, alcohol intake, BMI and waist circumference are shown in Table 2. We observed an improvement in: moderate-vigorous physical activity (150.7 minutes); TV viewing ( -1.4 hours/week); processed meat intake (-1.2 serves/week), fruit (0.3 serves/day) and vegetable intake (1.0 serve/day), BMI $\left(-1.4 \mathrm{~kg} / \mathrm{m}^{2}\right)$ and waist circumference $(-5.1 \mathrm{~cm})$. At baseline, 7 participants were former smokers and 1 participant was a current smoker. The median number of cigarettes smoked by the former and current smokers was 14 (range $3-30$ ) with the median starting age of 18 years (range 10-25). During the intervention period, the 1 current smoker quit smoking. Further, from baseline to 6 weeks, participants were more likely to meet the Australian recommendations for moderate-vigorous physical activity (27.3 to 59.1\%), red meat (86.4 to $90.9 \%$ ), fruit ( 68.2 to $81.8 \%$ ),vegetables (4.6 to $18.2 \%$ ), alcohol (59.1 to $72.7 \%$ ), BMI (31.8 to $45.5 \%$ ) and waist circumference (18.2 to $27.3 \%$; Table 3 ).

\section{HRQoL}

From baseline to 6 weeks there was an improvement in physical (3.3) and mental HRQoL scores (4.4). We also observed improvements in physical functioning (2.2), bodily pain (5.8), general health (3.7), vitality (4.8); and role-physical (3.1), social functioning (4.3), roleemotional (3.7) and mental health (4.8; Table 4).

\section{CRC screening and perceived risk of CRC}

At baseline 14 participants had been screened for CRC, while an additional 2 participants were screened during the intervention period. From baseline to 6 weeks [baseline \% (SD) vs 6 weeks \% (SD), $p$ value], we observed an 
Table 2 Mean change in moderate to vigorous physical activity (MVPA), TV viewing, diet (red meat, processed meat, fruit, vegetables), alcohol, body mass index and waist circumference from baseline to six weeks

\begin{tabular}{|c|c|c|c|c|}
\hline Variable & Baseline mean (SD) & Six weeks mean (SD) & Six weeks - baseline $(95 \% \mathrm{Cl})$ & $p$ \\
\hline MVPA, mins/week & $66.6(21.1)$ & $217.3(34.7)$ & $150.7(22.7110 .5)$ & $<0.01$ \\
\hline TV viewing, hrs/week & $12.9(1.7)$ & $11.5(1.6)$ & $-1.4\left(\begin{array}{lll}-4.0 & 1.2\end{array}\right)$ & 0.28 \\
\hline \multicolumn{5}{|l|}{ Diet } \\
\hline Red meat, serves/week & $2.8(1.5)$ & $2.8(1.5)$ & $0.02(-0.60 .6)$ & 0.93 \\
\hline Processed meat, serves/week & $2.0(1.8)$ & $0.9(1.2)$ & $-1.2(-1.8-0.5)$ & $<0.01$ \\
\hline Fruit, serves/day & $1.8(0.9)$ & $2.2(1.2)$ & $0.3(-0.30 .9)$ & 0.27 \\
\hline Vegetables, serves/day & $2.5(1.3)$ & $3.5(1.1)$ & $1.0(0.41 .6)$ & $<0.01$ \\
\hline Alcohol, standard drinks/day & $1.9(0.3)$ & $1.5(0.3)$ & $-0.4(-0.80 .1)$ & 0.13 \\
\hline Body mass index, $\mathrm{kg} / \mathrm{m}^{2}$ & $30.4(7.4)$ & $29.0(6.1)$ & $-1.4(-2.3-0.5)$ & $<0.01$ \\
\hline Waist circumference, cm & $97.5(18.4)$ & $92.4(15.7)$ & $-5.1(-8.3-2.0)$ & $<0.01$ \\
\hline
\end{tabular}

increase in the proportion of participants who believed that a diagnosis of CRC was related to family history [27 (23.9) vs 31.8 (21.6), $p=0.52$ ], and their perceived risk of developing CRC in their lifetime decreased [49.3 (27.0) vs 39.4 (23.3), $p=0.20$ ].

Table 3 Proportion of participants meeting the national recommendations for health behaviours at baseline and follow up

\begin{tabular}{|c|c|c|c|}
\hline Variable & Baseline n (\%) & Six weeks n (\%) & $p$ \\
\hline \multicolumn{4}{|l|}{$\mathrm{MVPA}^{1}$} \\
\hline$\geq 150$ mins/week & $6(27.3)$ & $13(59.1)$ & \\
\hline$<150$ mins/week & 16(72.7) & $9(40.9)$ & 0.02 \\
\hline \multicolumn{4}{|l|}{ Red Meat } \\
\hline$\leq 4$ serves/week & $19(86.4)$ & 20(90.9) & \\
\hline$>4$ serves/week & $3(13.6)$ & $2(9.1)$ & 0.12 \\
\hline \multicolumn{4}{|l|}{ Fruit } \\
\hline$\geq 2$ serves/day & $15(68.2)$ & 18(81.8) & \\
\hline$<2$ serves/day & $7(31.8)$ & $4(18.2)$ & 0.04 \\
\hline \multicolumn{4}{|l|}{ Vegetables } \\
\hline$\geq 5$ serves/day & $1(4.6)$ & $4(18.2)$ & \\
\hline$<5$ serves/day & $21(95.5)$ & 18(81.8) & 0.03 \\
\hline \multicolumn{4}{|l|}{ Alcohol } \\
\hline$\leq 2$ standard drinks/day & 13(59.1) & 16(72.7) & \\
\hline$>2$ standard drinks/day & $9(40.9)$ & $6(27.3)$ & $<0.01$ \\
\hline \multicolumn{4}{|l|}{ Body mass index } \\
\hline Normal weight ${ }^{2}$ & $7(31.8)$ & $10(45.5)$ & \\
\hline Overweight/obese $^{3}$ & $15(68.2)$ & $12(54.5)$ & $<0.01$ \\
\hline \multicolumn{4}{|l|}{ Waist circumference } \\
\hline Low risk ${ }^{4}$ & $4(18.2)$ & $6(27.3)$ & \\
\hline Increased risk ${ }^{5}$ & 18(81.8) & $16(72.7)$ & $<0.01$ \\
\hline
\end{tabular}

Moderate to vigorous physical activity. 2. Normal weight $(18.5-24.9 \mathrm{~kg} / \mathrm{m} 2)$. 3. Overweight/obese ( $\geq 25 \mathrm{~kg} / \mathrm{m} 2)$. 4 . Low risk ( $\leq 94 \mathrm{~cm}$ men, $\leq 80 \mathrm{~cm}$ women). 5. Increased risk ( $>94 \mathrm{~cm}$ men, $>80 \mathrm{~cm}$ women).

\section{Program satisfaction}

$100 \%$ of participants were highly satisfied with the intervention overall, $74 \%$ were highly satisfied with the health coaches, $89 \%$ were highly satisfied with the CanPrevent handbook, and $100 \%$ stated that they would recommend the intervention to others at risk of CRC.

\section{Discussion}

This report describes the acceptability and short-term effectiveness of CanPrevent, a telephone-delivered theory-based intervention to improve health behaviours for first degree relatives of CRC survivors. We received an overwhelmingly positive response from potential participants with a $100 \%$ intervention retention rate. Participants also reported that the intervention was highly acceptable and that they would all recommend it to others at risk of CRC. From baseline to six weeks, we observed improvements in moderate-vigorous physical activity, TV viewing, processed meat intake, fruit and vegetable intake, BMI and waist circumference. and the only current smoker at baseline quit during the intervention. Further, participants were more likely to meet the national recommendations for moderate-vigorous physical activity, fruit, vegetable and alcohol intake, BMI and waist circumference. Participants reported an improvement in the SF-36 summary scores (physical and mental HRQoL), as well as for the SF-36 subscales (rolephysical, bodily pain, general health, vitality, social functioning, role-emotional and mental health). Finally, at six weeks participants were more likely to believe a diagnosis of CRC was related to family history. There was also a decrease in their perceived risk of developing CRC in their lifetime following participation in CanPrevent, highlighting the importance of investigating beliefs regarding lifestyle factors and perceived risk of CRC.

With the limitations of a small acceptability and shortterm effectiveness study in mind, the CanPrevent intervention results were very positive compared with the 
Table 4 Mean change in health-related quality of life (HRQOL) from baseline to six weeks

\begin{tabular}{|c|c|c|c|c|}
\hline Short form-36 subscale & Baseline mean (SD) & Six weeks mean (SD) & Six weeks - baseline $(95 \% \mathrm{Cl})$ & $p$ \\
\hline Physical Functioning & $50.6(6.5)$ & $52.8(4.4)$ & $2.2(0.93 .5)$ & $<0.01$ \\
\hline Role Physical & $47.2(8.5)$ & $50.2(7.7)$ & $3.1(-0.97 .0)$ & 0.12 \\
\hline Bodily Pain & $45.0(11.4)$ & $50.7(9.7)$ & $5.8(1.410 .1)$ & 0.01 \\
\hline General Health & $48.4(29.6)$ & $52.1(8.6)$ & $3.7(0.37 .1)$ & 0.03 \\
\hline Vitality & $47.3(42.7)$ & $52.0(49.3)$ & $4.8(1.48 .2)$ & 0.01 \\
\hline Social Functioning & $48.0(43.2)$ & $52.3(49.8)$ & $4.3(-0.49 .0)$ & 0.07 \\
\hline Role Emotional & $47.0(14.2)$ & $50.7(6.5)$ & $3.7(-2.09 .4)$ & 0.19 \\
\hline Mental Health & $45.8(13.7)$ & $50.6(6.8)$ & $4.8(-0.7$ 10.3) & 0.08 \\
\hline Physical HRQoL & $48.6(1.6)$ & $51.9(6.6)$ & 3.3 (03 6.3) & 0.03 \\
\hline Mental HRQoL & $46.4(14.9)$ & $50.8(6.9)$ & $4.4(-1.9$ 10.7) & 0.16 \\
\hline
\end{tabular}

findings of previous investigators [20,21]. We observed improvements in multiple risk factors as well as HRQoL. It is also important to note that the improvements in HRQoL are considered clinically significant (2-5 point change) [61]. In comparison, PREVENT investigators observed significant intervention effects for multivitamin and red meat intake [21] and Bowel Health to Better Health had significant intervention effects on fibre intake alone [20]. The current study participants were also more likely to meet the national recommendations for most health behaviours post-intervention. These positive findings may be attributed to the fact that CanPrevent focused on multiple health behaviours which may have maximised the intervention effect. Further, CanPrevent was a novel ACT-based intervention that used strategies to overcome internal barriers to behavioural improvements by emphasizing the role of emotions and thoughts in the maintenance of good self-management of health behaviours. Consistent with the literature, it is possible that higher levels of physical activity in particular during participation in CanPrevent may have contributed to the observed improvements in HRQoL as physical activity has direct physical and mental health benefits [66]. Whilst the observed improvement in social functioning may have been a result of the regular telephone contact with the health coach during the intervention period. However, further research is required to confirm the positive findings of this short-term effectiveness trial, to identify mediators of the intervention effects, and to determine whether the observed improvements in health behaviours and HRQoL can be sustained.

There were a number of strengths to this study including: validated and reliable outcome measures that have been used over the telephone; a theory-based multiple behavior change intervention; a high rate of intervention delivery; a potentially low-cost, high-reach intervention; and a high level of interest and satisfaction with the intervention. Importantly, mediated (non-face-to-face) intervention delivery can be cost-effective and provide repeated contacts necessary to promote behavior change [67]. Telephone delivery is one of the most accessible mediated approaches and has potential for adoption by organisations that routinely operate telephone information and support centres [67]. The study was limited by use of self-report measures and their inherent biases, although all measures have been routinely used in population-based epidemiological and intervention research and over the telephone. Data were also collected by telephone interview which limited our ability to collect objective biomedical data. There were also more female than male participants, and the small sample size and lack of a control group were significant limitations. However this small pilot study was primarily designed to test the acceptability and short term effectiveness of the CanPrevent intervention.

\section{Conclusions}

This study provides further support that comprehensive interventions focusing on a range of health behaviours can result in improvements in health outcomes. CanPrevent was acceptable and the results of this study suggest that the intervention may be effective in promoting multiple health behaviour, and HRQoL, improvements. A larger scale randomised controlled trial is required to confirm these findings and to determine longer term effectiveness.

\section{Abbreviations}

CRC: Colorectal cancer; HRQoL: Health-related quality of life; MVPA: Moderate to vigorous physical activity.

\section{Competing interests}

The authors declare that they have no competing interests.

\section{Authors' contributions}

ALH initiated the study and developed the study protocol. JFA and AG contributed to the final study protocol and study materials. TAP was responsible for implementing the study protocol. ALH drafted the manuscript and all authors contributed to the final version. All authors read and approved the final manuscript. 


\section{Acknowledgements}

This study was funded by the Cancer Council Queensland. We gratefully acknowledge the input from Ms Rhianna Hardie, Ms Susan Bell, Ms Bernice Kelly and Ms Sarah Mitchell in the development and delivery of the intervention, and Ms Marnie Dunn in the collection of data.

\section{Author details}

${ }^{1}$ School of Public Health and Social Work, Queensland University of Technology, Victoria Park Road, Kelvin Grove, Brisbane, Queensland 4059, Australia. ${ }^{2}$ Viertel Centre for Research in Cancer Control, Cancer Council Queensland, PO Box 201, Spring Hill, Brisbane, Queensland 4004, Australia. ${ }^{3}$ The University of Queensland Health Service, The University of Queensland, Brisbane, Australia.

Received: 2 April 2012 Accepted: 22 November 2012 Published: 27 November 2012

\section{References}

1. Ferlay J, Bray F, Pisani P, Parkin DM: GLOBOCAN 2002: Cancer Incidence. Mortality and Prevalence Worldwide. Lyon: IARC Press; 2004.

2. Australian Institute of Health and Welfare: Cancer Incidence Projections Australia, 2002 to 2011. Canberra: Australian Institute of Health and Welfare; 2005

3. Baade P, Fritschi L, Eakin E: Non-cancer mortality among people diagnosed with cancer (Australia). Cancer Causes Control 2006, 17:287-297.

4. Brown BW, Brauner C, Minnotte MC: Noncancer deaths in white adult cancer patients. J Nat/Cancer Inst 1993, 85:979-987.

5. Samad AK, Taylor RS, Marshall T, Chapman MA: A meta-analysis of the association of physical activity with reduced risk of colorectal cancer. Colorectal Dis 2005, 7:204-213.

6. Gonzalez CA: Nutrition and cancer: the current epidemiological evidence. Br J Nutr 2006, 96(Suppl 1):S42-45.

7. Terry P, Giovannucci E, Michels KB, Bergkvist L, Hansen H, Holmberg L, Wolk A: Fruit, vegetables, dietary fiber, and risk of colorectal cancer. J Natl Cancer Inst 2001, 93:525-533.

8. Bingham SA, Day NE, Luben R, Ferrari P, Slimani N, Norat T, Clavel-Chapelon $F$, Kesse $E$, Nieters $A$, Boeing $H$, et al: Dietary fibre in food and protection against colorectal cancer in the European Prospective Investigation into Cancer and Nutrition (EPIC): an observational study. Lancet 2003, 361:1496-1501.

9. Bianchini F, Kaaks R, Vainio H: Overweight, obesity, and cancer risk. Lancet Oncol 2002, 3:565-574

10. Coyle YM: Lifestyle, genes, and cancer. Methods Mol Biol 2009, 472:25-56.

11. Moghaddam AA, Woodward M, Huxley R: Obesity and risk of colorectal cancer: a meta-analysis of 31 studies with 70,000 events. Cancer Epidemiol Biomarkers Prev 2007, 16:2533-2547.

12. Platz EA, Willett WC, Colditz GA, Rimm EB, Spiegelman D, Giovannucci E: Proportion of colon cancer risk that might be preventable in a cohort of middle-aged US men. Cancer Causes Control 2000, 11:579-588.

13. Australian Institute of Health and Welfare: Chronic Disease and Associated Risk Factors, 2001. In Chronic Disease and Associated Risk Factors, 2001. Canberra: AlHW; 2002.

14. Australian Institute of Health and Welfare, Bowel Cancer Screening Pilot Monitoring and Evaluation Steering Committee: Australia's Bowel Cancer Screening Pilot and Beyond FINAL EVALUATION REPORT. Canberra: Commonwealth of Australia; 2005.

15. Johns LE, Houlston RS: A systematic review and meta-analysis of familial colorectal cancer risk. Am J Gastroenterol 2001, 96:2992-3003.

16. Marchand LL: Combined influence of genetic and dietary factors on colorectal cancer incidence in Japanese Americans. J Natl Cancer Inst Monogr 1999, 26:101-105.

17. McCaffery K, Wardle J, Waller J: Knowledge, attitudes, and behavioral intentions in relation to the early detection of colorectal cancer in the United Kingdom. Prev Med 2003, 36:525-535.

18. Almendingen $\mathrm{K}$, Hofstad $\mathrm{B}$, Vatn $\mathrm{MH}$ : Lifestyle-related factors and colorectal polyps: preliminary results from a Norwegian follow-up and intervention study. Eur J Cancer Prev 2002, 11:153-158.

19. Blalock SJ, DeVellis BM, Afifi RA, Sandler RS: Risk perceptions and participation in colorectal cancer screening. Health Psychol 1990, 9:792-806.
20. Caswell S, Anderson AS, Steele RJ: Bowel health to better health: a minimal contact lifestyle intervention for people at increased risk of colorectal cancer. Br J Nutr 2009, 102(11):1541-1546.

21. Emmons KM, McBride CM, Puleo E, Pollak Kl, Clipp E, Kuntz K, Marcus BH, Napolitano M, Onken J, Farraye F, Fletcher R: Project PREVENT: a randomized trial to reduce multiple behavioral risk factors for colon cancer. Cancer Epidemiol Biomarkers Prev 2005, 14:1453-1459.

22. Bandura A: Social foundations of thought and action: a social cognitive theory. Englewood Cliffs: Prentice-Hall; 1986.

23. King TK, Marcus BH, Pinto BM, Emmons KM, Abrams DB: Cognitivebehavioral mediators of changing multiple behaviors: smoking and a sedentary lifestyle. Prev Med 1996, 25:684-691.

24. Emmons KM, Marcus BH, Linnan L, Rossi JS, Abrams DB: Mechanisms in multiple risk factor interventions: smoking, physical activity, and dietary fat intake among manufacturing workers. Working Well Research Group. Prev Med 1994, 23:481-489.

25. Blair SN, Jacobs DR Jr, Powell KE: Relationships between exercise or physical activity and other health behaviors. Public Health Rep 1985, 100:172-180.

26. Unger JB: Stages of change of smoking cessation: relationships with other health behaviors. Am J Prev Med 1996, 12:134-138.

27. Painter JE, Borba CP, Hynes M, Mays D, Glanz K: The use of theory in health behavior research from 2000 to 2005: a systematic review. Ann Behav Med 2008, 35:358-362.

28. Hayes SC: Acceptance and commitment therapy, rational frame therapy, and the third wave of behavioral and cognitive therapies. Behav Ther 2004, 35:639-665.

29. Hayes SC, Masuda A, Bissett R, Luoma JB, Guerrero LF: DBT, FAP and ACT: How empirically orientated are the new behaviour therapy technologies? Behav Ther 2004, 35:35-54.

30. Hayes SC, Strosahl KD, Wilson KG: Acceptance and Commitment therapy: An experimential approach to behavior change. New York: The Guilford Press; 1999.

31. Hayes SC, Luoma JB, Bond FW, Masuda A, Lillis J: Acceptance and commitment therapy: Model, processes and outcomes. Behavioural Research and Therapy 2006, 44:1-25.

32. Henry JL, Wilson PH, Bruce DG, Chisholm DJ, Rawling PJ: Cognitivebehavioural stress management for patients with noninsulin dependent diabetes mellitus. Psychol Health Med 1997, 2:109-118.

33. Dahl J, Wilson KG, Nilsson A: Acceptance and committment therapy and the treatment of persons at risk for long-term disability resulting from stress and pain symptoms: a preliminary randomized trial. Behav Ther 2004, 35:785-801.

34. Gregg JA, Callaghan GM, Hayes SC, Glenn-Lawson JL: Improving diabetes self-management through acceptance, mindfulness and values: a randomised controlled trial. J Consult Clin Psychol 2007, 75:336-343.

35. Lundgren TA, Dahl J, Melin L, Kies B: Evaluation of accepatance and committment therapy for drug refractory epilepsy: a randomised controlled trial in South America: a pilot study. Epilepsia 2006, 47:2173-2179

36. Gifford EV, Kohlenberg BS, Hayes SC, Antonuccio DO, Piasecki MM, Rasussen-Hall ML, Palm KM: Acceptance-based treatment for smoking cessation. An initial trial of Acceptance and Committment Therapy. Behav Ther 2004, 35:689-705

37. Forman EM, Butryn $\mathrm{M}$, Hoffman $\mathrm{KL}$, Herbert JD: An open trial of an acceptance-based behavioral treatment for weight loss (PDF). Cognitive and Behavioral Practice 2009, 16:223-235.

38. Forman EM, Hoffman KL, McGrath KB, Herbert JD, Brandsma LL, Lowe MR: A comparison of acceptance- and control-based strategies for coping with food cravings: an analog study. Behav Res Ther 2007, 45:2372-2386.

39. Lillis J, Hayes SC, Bunting K, Masuda A: Teaching acceptance and mindfulness to improve the lives of the obese: a preliminary test of a theoretical model. Ann Behav Med 2009, 37:58-69.

40. Mishel MH, Belyea M, Germino BB, Stewart JL, Bailey DE Jr, Robertson C, Mohler J: Helping patients with localized prostate carcinoma manage uncertainty and treatment side effects: nurse-delivered psychoeducational intervention over the telephone. Cancer 2002, 94:1854-1866.

41. Stull VB, Snyder DC, Demark-Wahnefried W: Lifestyle interventions in cancer survivors: designing programs that meet the needs of this vulnerable and growing population. J Nutr 2007, 137:243S-248S. 
42. Morey MC, Snyder DC, Sloane R, Cohen HJ, Peterson B, Hartman TJ, Miller P, Mitchell DC, Demark-Wahnefried W: Effects of home-based diet and exercise on functional outcomes among older, overweight long-term cancer survivors. JAMA 2009, 301:1883-1891.

43. Eakin E, Reeves M, Lawler S, Graves N, Oldenburg B, Del Mar C, Wilke K, Winkler E, Barnett A: Telephone counseling for physical activity and diet in primary care patients. Am J Prev Med 2009, 36:142-149.

44. Eakin EG, Lawler SP, Vandelanotte C, Owen N: Telephone interventions for physical activity and dietary behavior change: a systematic review. Am J Prev Med 2007, 32:419-434

45. Australian Bureau of Statistics: Household telephone connections. Queensland: Australian Bureau of Statistics, Australian Government; 2003. Cat No. 8159.3; 2003.

46. Department of Health and Aged Care: National Physical Activity Guidelines for Australians. Canberra: Australian Government; 1999.

47. Department of Health and Ageing: National Health and Medical Research Council. Food For Health. Dietary Guidelines for Australians. A Guide to Healthy Eating. In Book National Health and Medical Research Council. Food For Health. Dietary Guidelines for Australians. A Guide to Healthy Eating: Australian Government; 2005.

48. Promoting Healthy Weight: Promoting Healthy Weight. http://www.health. gov.au/internet/main/publishing.nsf/Content/health-pubhlth-strateg-hlthwtindex.htm.

49. National Health and Medical Research Council: Australian Alcohol Guidelines: Health Risks and Benefits. Australian Alcohol Guidelines: Health Risks and Benefits, Canberra 2001.

50. Hawkes AL, Chambers SK, Pakenham KI, Patrao TA, Baade P, Lynch BM, Aitken JF, Meng X, Courneya KS: Effects of a telephone-delivered multiple health behavior change intervention on health and behavioral outcomes in colorectal cancer survivors ('CanChange'): A randomized controlled trial. J Clin Oncol, (submitted August 2012).

51. Hawkes AL, Gollschewski S, Lynch BM, Chambers S: A telephone-delivered lifestyle intervention for colorectal cancer survivors 'CanChange': a pilot study. Psychooncology 2009, 18:449-455.

52. Hawkes AL, Pakenham Kl, Courneya KS, Gollschewski S, Baade P, Gordon LG, Lynch BM, Aitken JF, Chambers SK: A randomised controlled trial of a telebased lifestyle intervention for colorectal cancer survivors ('CanChange'): study protocol. BMC Cancer 2009, 9:286.

53. Godin G, Shephard RJ: A simple method to assess exercise behaviour in the community. Can J App/ Sports Science 1985, 10:141-146.

54. Clark BK, Sugiyama T, Healy GN, Salmon J, Dunstan DW, Owen N: Validity and reliability of measures of television viewing time and other nonoccupational sedentary behaviour of adults: a review. Obes Rev 2009, 10:7-16.

55. Salmon J, Owen N, Crawford D, Bauman A, Sallis JF: Physical activity and sedentary behaviour: a population-based study of barriers, enjoyment and preference. Health Psychol 2003, 22:178-188.

56. The Cancer Council New South Wales: Cancer-related knowledge and practices: recommended survey items. Sydney: Cancer Council New South Wales; 2006.

57. Department of Health and Ageing, National Health and Medical Research Council: Food For Health. Dietary Guidelines for Australians. A Guide to Healthy Eating. Canberra: Australian Government; 2005.

58. International agency for Cancer on Research: Weight control and physical activity. IARC Handbooks of Cancer Prevention: IARC; 2002.

59. Rimm EB, Stampfer MJ, Colditz GA, Chute CG, Litin LB, Willett WC: Validity of self-reported waist and hip circumferences in men and women. Epidemiology 1990, 1:466-473.

60. National Health and Medical Research Council: Clinical Practive Guidelines for the Management of Overweight and Obesity in Adults, Canberra, Australia; 2003.

61. Ware J, Kosinski M, Keller S: SF 36 Physical and Mental Health Summary Scales: A User's Manual. MA: The Health Institute, New England Medical Centre; 1994.

62. Ware J, Sherbourne C: The MOS 36-item short-form health survey (SF-36). Conceptual framework and item selection. Med Care 1992, 30:473-483.

63. Australian Cancer Network Colorectal Cancer Guidelines Revision Committee: Guidelines for the Prevention, Early Detection and Management of Colorectal Cancer. Canberra: The Cancer Council Australia and Australian Cancer Network; 2005
64. Department of Health and Ageing: National Tobacco Strategy, 2004-2009: The Strategy, Canberra, Australia; 2005.

65. Tudor-Locke C, Bassett DR Jr: How many steps/day are enough? Preliminary pedometer indices for public health. Sports Med 2004, 34:1-8.

66. Fletcher GF, Balady G, Blair SN, Blumenthal J, Caspersen C, Chaitman B, Epstein S, Froelicher ESS, Froelicher VF, Pina IL, Pollock ML: Statement on exercise: benefits and recommendations for physical activity programs for all Americans: a statement for health professionals by the committee on exercise and cardiac rehabilitation of the council on clinical cardiology, American Heart Association. Circulation 1996, 94:857-862.

67. Goode AD, Reeves MM, Eakin EG: Telephone-delivered interventions for physical activity and dietary behavior change: an updated systematic review. Am J Prev Med 2012, 42:81-88.

doi:10.1186/1471-2407-12-560

Cite this article as: Hawkes et al:: CanPrevent: a telephone-delivered intervention to reduce multiple behavioural risk factors for colorectal cancer. BMC Cancer 2012 12:560.

\section{Submit your next manuscript to BioMed Central and take full advantage of:}

- Convenient online submission

- Thorough peer review

- No space constraints or color figure charges

- Immediate publication on acceptance

- Inclusion in PubMed, CAS, Scopus and Google Scholar

- Research which is freely available for redistribution 